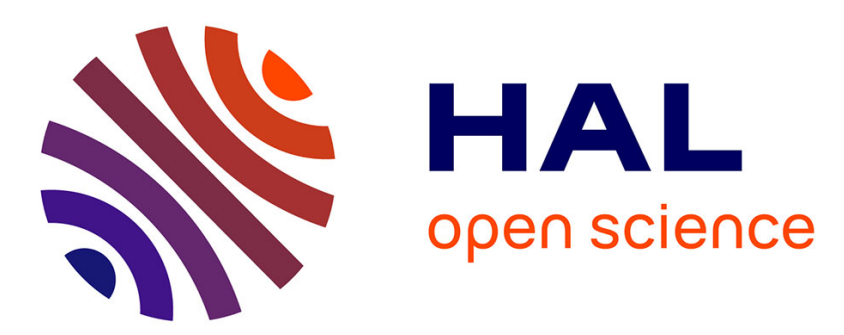

\title{
Web Ontologies as Renewal of Classical Philosophical Ontology
}

Pierre Livet

\section{To cite this version:}

Pierre Livet. Web Ontologies as Renewal of Classical Philosophical Ontology. Metaphilosophy, 2012, 43, pp.396 - 404. 10.1111/j.1467-9973.2012.01757.x . hal-01079850

\section{HAL Id: hal-01079850 \\ https://hal.science/hal-01079850}

Submitted on 3 Nov 2014

HAL is a multi-disciplinary open access archive for the deposit and dissemination of scientific research documents, whether they are published or not. The documents may come from teaching and research institutions in France or abroad, or from public or private research centers.
L'archive ouverte pluridisciplinaire HAL, est destinée au dépôt et à la diffusion de documents scientifiques de niveau recherche, publiés ou non, émanant des établissements d'enseignement et de recherche français ou étrangers, des laboratoires publics ou privés. 


\title{
WEB ONTOLOGIES AS RENEWAL OF CLASSICAL PHILOSOPHICAL ONTOLOGY PIERRE LIVET \\ CEPERC \\ University of Aix-Marseille
}

\begin{abstract}
Do Web devices (addresses, tags, networks, and the rest) have counterparts in classical ontology? Yes, but they allow us also to introduce more refined distinctions. In addition, their dynamic use could inspire a dynamic reconception of classical ontology. In the process of making explicit ontological types, different types can be undistinguished as first steps (considered as "floating types") to be defined only in a further step, one in which their function as distinguishers of other kinds of entities has to be made explicit. The fact that the ontological import of some node in a network of tags and addresses could evolve with the transformation of the network can most easily find an interpretation in this dynamic conception of ontology.
\end{abstract}

Keywords: ontology, WEB, referent, URL, types.

\section{Introduction}

Philosophical ontologies, when they are seen as giving a definition of the fundamental types of entities that there are in our world - and in possible worlds - are nowadays mixtures of Fregean and either Aristotelian or Ockhamian conceptions. Everybody has in mind the Fregean distinction between reference (Bedeutung) and meaning or signification (Sinn), and their possible relation with argument and function in logic and mathematics (Frege 1984). Fundamental ontological entities have to be coordinated with that duality. In an updated Aristotelian tradition, substances taken as substrates (particular substrates, since universal substances are defended only by a few philosophers) are the targets of the operation of reference, and properties (universal and particular ones) are the ontological counterparts of predicates, themselves partially related to functions (Grenon 2003). In a similarly renewed Ockhamian tradition (the so-called tropist current), referents are identified by the compresence of particular qualities or concrete properties, and signification is related to similarities between particular qualities or properties. In a more classical nominalist current, reference is made to particular substrates, and signification implies the contribution of particular properties, universal properties being excluded (Livet and Nef 2009).

At first sight, present Web ontologies seem to be combinations of addresses (URI, URL) and names of classes in different domains, with the help of some basic relations like "has a link with," "is a ...," and maybe "is a part of ..." that make it possible to build networks representing the ontology of a domain, and the articulations between different domains (Smith 2008). Addresses could be seen as the correspondents of referents, and names as the correspondents of significations.

In what follows, I suggest that upon closer examination we discover that Web ontologies introduce more subtle distinctions - at least a triadic distinction instead of a dualistic one. I also suggest that we have to conceive Web ontologies dynamically. In the dynamics of the construction and the evolution of Web ontological networks, the ontological type of an item will not really remain fixed but can change in relation to its interactions with the transformation of the structure of the network. In a sense, everything in Web ontology is an address, and the difference between types and addresses (between classes of content and references) is only given by different modes of cross-relation: chains of relations between addresses, and transversal relations across these chains for types. The mode of structuration is more fundamental than the kinds of entities. This relational and dynamic approach has to be taken more seriously by philosophical ontologies, which are usually centred on static entities. In this dynamic perspective, the old dualities of philosophical ontology could be reduced to the duality of two operations: distinguishing items - trying to identify them, even only relatively — and making them and their articulations explicit. For the sake of brevity, I will use the neologism "explicitation" instead of "making explicit." Explicitation is a process that starts with a basic operation of distinction that distinguishes items that were previously undistinguished and proceeds by also using other previously undistinguished items in order to make explicit the articulations between the distinguished items. The definition of the type of the entities is then relative to the stage of the process of explicitation. Because distinctions are coarse-grained ones at the beginning of the processes, several kinds of entities are still not distinguished at this stage (for example, the considered items could indifferently be a particular substrate, a particular quality, or even a universal property). A specific ontological type of entity is defined in relation to the stage at which it has been necessary to distinguish it from other previous ontological types of entities. Up to this stage, the ontological type of an entity can be considered a "floating type,"floating between the different types that are still possible.

In the Web the ontology and its explicitation are conditional on the process of developing the network and are related to the different stages of this process, considered a recursive one. In this sense, the Semantic Web

(Web 3.0) - the kind of Web for which ontologies are required, even if they are also useful for the knowledge Web (Web 4.0) — could retain somefeatures of the social Web (Web 2.0) in which folksonomies emerge fromthe 
free tagging of information and objects by users when one tries to relate these tags to each other. This dynamics of recursively making explicit floating types seems to be a fundamental property of the ontologies of Web 2.0, 3.0, and 4.0, and could also be a property of a philosophical ontology that includes in it the ontology of its own processes of explicitation.

\section{Addresses, Reference, and Signification}

Addresses on the Web seem just to be pointers to locations. As pointers, they surely have the function of referring to items. Tags or names seem to have the function of pointing out meaningful content. Accordingly, they seem to be candidates for the function of signifiers in the domain of thought and for predicates in a logical language. Addresses and tags seem to correspond to the two main Fregean categories.

Unfortunately (or, I as will suggest, fortunately), this is far too simple. First, in the social Web, tags belong to folksonomies. If you want to move from those folksonomies to the Semantic Web, you have to solve the difficult problem of how to homogenize the different contents to which the tags refer. If you are successful in doing so, you have given up the functions of tags that were not reducible to conceptual contents: for example, contextual connotations and marks of reference to the group of the users of these tags. A way of saving a part of those very diverse functions is to take tags as labels for nodes in a network: at least some of the contextual functions will be kept. In this case, tags are no longer reducible to Fregeansignifications, for those are not context sensitive. Since pointing out nodes in a network is also a referential function, tags keep some referential function, but now this function is contextualized.

Similar complications occur for addresses. We could believe that addresses point to nodes, which are locations in the structure of the network. The problem is that any change in the network changes the structure of its relations and so also the relational role or the nodes. Some new nodes become accessible if you add links and of course new added nodes are reachable by these new links. As there is no previously existing homogenous structure of locations, no space pre-existing the nodes and the links of the network, the structure of the network space is changed each time new nodes and new links are added—or old nodes and old links are erased.

In a sense, this network structure makes both addresses and tags (as related to nodes) more similar to inferential paths. The inferential content of a conceptual term (for Frege a conceptual term is the counterpart of a signification in the realm of thought) is determined by all the paths of inference in which it participates. If you change the tree or the network of those paths, you change the meaning of the concept. Frege would have replied that even if conceptual representations and processes of psycho- logical inference could evolve, the significations are stable. But adding new links is not only to add newly discovered but stable significations, it can in addition change the contextual import of the previous ones. The ontology of the Web has to take into account this possibility.

All this would be true even if addresses were only ways of pointing out locations, in a network that is not a homogenous and pre-existing structure but a structure that is constantly rebuilt. But addresses are not reducible to that function alone. They give access to resources. Giving such access is not only to point towards the resource. Giving access is already to use the resource as such. If giving access is considered a function, using the resource is in a sense a function (a function of the function of giving access). We are far from the strict notion of referent. Or, to take things another way, we discover that having access to a referent is much more than pointing it out.

Things are even more complicated. Monnin (2012) has drawn my attention to the fact that "resources" do not always give access to the same content (if the content is updated every day, for example). In this respect they behave more like indexicals, but in contrast to indexicals, they give you some access to the content (if there is one) without requiring additional information. In addition, an address is not just a URL (uniform resource locator) but more generally a URI (uniform resource identifier).

URIs just give the name of a resource, independent of its location and the way in which is it referenced. In a sense, URIs could have been taken as the real Fregean referents, because Frege takes them as referents independently of any particular way of referencing. But from another perspective,

URIs are the names of the referents, as they are not what is there in the indicated location. Are they proper names or common ones? They could be seen as the common names (common to different ways of referencing) of proper names. Here again the ontological resources of the Web ontologies pass the Fregean distinctions by recombining features of their different categories.

Another interesting distinction of Web ontologies is the difference between the closed world assumption and the open world assumption. The open world assumption implies the possibility that our ontology is incomplete. New ontological types could need to be added in the future, contrary to the requirement of the closed world assumption (according to which all types of entities have already been spelled out). Since reference is now related to access, this distinction enriches the list of the different functions of referring. We can refer to an accessible referent by its proper name, knowing its location. We can refer to an accessible referent without knowing its location, but being able to name it and to put it on our list of referred entities. Eventually we can refer "out of the blue" to referents forwhich we have neither location nor name. They are only supposed to 
beaccessible if we can extend our world, or go beyond the present world to which we currently have access. The notion of "referent" can be taken in each of these three ways.

Apparently, this is not possible for significations. We have no real common names for entities of the third category, entities to which we currently have no access at our disposal. We indicate them by using paraphrases and what could be better called "indefinite" descriptions ("one of the entities to which we do not currently have access at our disposal"). Only a dualist distinction seems possible: significations can be accessible by common names, without a real definition of the precise content of the common name (only a nominal one), or by their conceptual content.

Is there a way to restore the symmetry between referents and significations, and to have three real distinctions for both? We could introduce the triadic distinction between (a) what is not distinguished, (b) what is distinguished but not made explicit, and (c) what is distinguished and made explicit. This distinction works both for referents and for significations. If "referent" is related to "distinguished," a referent "out of the blue" is "undistinguished," a distinguished referent is a referent to which we can point even without knowing its location; knowing this location implies relating it to other distinguished entities, and this consists in making its distinction explicit. In the same way, the signification of an entity that is not distinguished from other ones is "out of the blue"; the signification of an entity is distinguished but not made explicit when it can be captured and when the ways in which it distinguishes it from other types of entities (what we could call its distinguishers) are still not themselves distinguished; the signification of an entity is explicit when the ways of distinguishing it from other types of entities have themselves been distinguished.

Apparently this threefold distinction is an epistemic distinction, not an ontological one. But epistemic distinctions have ontological bases: the processes by which they proceed, and the structure of the reality that allows those processes to proceed. We could have entities the structure of which cannot allow us to distinguish them from other ones; entities the structure of which allows us to distinguish them from other entities but do not allow us to distinguish their distinguishers; and entities the distinguishers of which can themselves be distinguished.

\section{Floating Types and Recursive Process of Explicitation}

The triadic basis gives rise to a recursive process, when it is possible to distinguish entities by using other entities to distinguish them, then to make explicit the previous distinctions by distinguishing the distinguishers (using other distinguishers), then to make explicit the distinguisher by distinguishing these other distinguishers, and so on.

For example (if we use the classical types of ontological entities) substrates can distinguish themselves from other substrates: particular substrates use other particular substrates as their distinguishers. Particular qualities can use other particular qualities as distinguishers. But how can particular substrates be distinguished from particular qualities? If we say that substrates as such distinguish themselves from qualities as such, using qualities as distinguishers, this answer gives rise to another question: What are the features of substrates and qualities that distinguish them from one another? We could answer by using different kinds of relations or even new types of entities. For example, if "quality" - and not "substrate" - is the most basic category, the relation of compresence of qualities (a quality is compresent with another if they co-exist together in a bundle) builds a substrate with its qualities (at the same level as the qualities and the relation). If we admit a difference between two kinds of entities (substrates and qualities), we have introduced not only the relation of difference but also, at another level, kinds as types of new entities - or maybe universals.

We see that when in the first step we focus on substrates, we assume at least other substrates in order to distinguish one substrate, but those substrates, used as distinguishers, are not themselves distinguished. They are still undistinguished. In this step we have considered three possibilities: the first is that there are only substrates, the second is that we could have only qualities and one relation-if the so-called substrates require the compresence of qualities - and the third is that we have only relations - if we consider compresence and similarity (or difference) as more fundamental than their relata. In the second step (differentiating substrates from qualities, for example) we could have kinds or universals, or relations, or both.

Suppose that in a third step we have to make explicit as distinguishers what is the glue or connection articulating one substrate and its quality.

We have then to make explicit one distinguisher of this substrate and its quality, the relation between the substance and its quality. If the quality is a universal, the substrate exemplifies this universal, and the relation is one of exemplification. If the quality is a particular, we need in addition to the relation of compresence the relation of instantiation, as a particular compresence is an instantiation of the universal relation of compresence. If we characterize the ontological entities that we have introduced by the step in which they have been made explicit, we see that particulars as referents are entities of first step (we could use particular substrates or particular qualities instead, but then qualities as particulars would be used only as identifiers). Qualities differing by their type from substrates are then entities of the second step. Relations distinguished from qualities and 
substrates - here we need to use as distinguishers universals, a categorythat is still not made explicit - are entities of the third step. Probably kinds or universals, if we use them, are entities of fourth step, and so on.

Such a recursive development makes it possible to take into account from the beginning dynamic processes in ontology, instead of having to presuppose static entities defined once and for all. The development makesnew distinguishers accessible. Along the way, it allows new distinctions.

We progress from a very coarse-grained step toward other, finer-grained steps. In a sense, in a very first step, a zero step, we have only undistinguished entities. Then in step 1 we have particulars. We do not know whether they are substrates, qualities, relations, or exemplifications or instantiations of universals. In step 2 we distinguished among particulars qualities and substrates. Relations, if they are in use, are still not distinguished and will be in a third step.

Let us go back to Web ontologies. Our dynamic process is a presentation of ontology that is more natural than the classical static ontologies, and is needed in order to characterize the development of ontologies in the different types of Web. Addresses can be used at first in a coarse-grained way. In this first step there are distinguishers, but they themselves are still not distinguished: their different possible operations-finding the location, naming the referent, relating it to other nodes of the network, making possible associations and inferences, giving access to stable contents or to continuously updated contents - are still taken together and are not necessarily distinguished from one another. Tags are also distinguishers, needing the machinery of URIs and URLs in order to be related to one location and then to each other. They are distinguished from addresses since thnote also that different tags can refer to the same location. When different tags are attached to the same location, they work as particular qualities attached to the same substrate or related by compresence. But if one tag can be used in different locations without equivocity, it has the status of either a relation or a universal. Tags seem to be entities emerging in our second step. Of course, in order to make explicit how addresses, tags common to different addresses, and tags common to the same addresses are organized, we need to specify the relations that build the network and avoid equivocity. These relations are made explicit in a third step.

For sure, in order to build the network of addresses, Web engineers have had to define relations from the beginning. But if for these engineers those relations have been made explicit, they do not function on the Web as made explicit - on the contrary, for users they are the hidden and undistinguished part of the Web. Relations become distinguishable for the user only with the social Web and the Semantic Web. The ontological entities that the users of the Web can refer to, using the technical facilities of the Web, seem to follow a progression very similar to the development of the process of explicitation, the outline of which I have just presented.

The task of the engineers of the different kinds of Web is to build structures that allow the development of dynamics that can fit with this rather "natural" development of the explicitation of the kinds of ontological entities. This development is a progressive one; formally it can be recursive.

We can use a kind of entity as a distinguisher without making explicit what this kind is, and we do not make explicit a kind of entity except if we need to specify its conditions of use. Such an explicitation needs other but not yet made explicit kinds of entities, and so on.

\section{Conclusion: Points Still to Be Made Explicit}

I hope to have shown here that a conception of ontology is possible that presents a fundamentally dynamic aspect, more in tune with the dynamics of the extension and the emergence of different forms and functions of Web 2.0, 3.0, and 4.0. The condition for doing so is to take into account in the ontology the processes by which we distinguish entities that were undistinguished at first and then make explicit those distinctions by distinguishing the entities that we have only used as distinguishers in our first task.

In this process, the distinction between reference and signification could seem to have been blurred, because referents and significations are both distinguishers. But it is not really the case, for in our first step (when a substrate uses another substrate to be distinguished) we have used a distinction between entities that are supposed not to differ by their kind, so that this distinction between several substrates was only used to distinguish a particular referent. Significations are at stake in the further steps-with qualities, relations, and universals.

How is this distinction maintained in the operations of Web ontology? We have seen how addresses and tagsour candidates at the basic and coarse-grained levels (coarse-grained with respect to ontological sophistication) have cross-functions. Addresses allow chains of paths from one location to another, and tags can either subsume different nodes or specify different features of the same nodes-while these features can be also put together under the umbrella of a generic tag. The knowledge Web requires a more elaborated and sophisticated ontology, with relations and processes of inference, for example, but it could be regarded as taking place in the same overall dynamic process of recursive ontological explicitation.

The parallel between this ontological development and the new functions built into the Web could lead us to define the canonical ways of such a development and to give in this way ontological landmarks that would useful for comparing things and avoiding becoming lost in the complexity of the Web. But such a reflexive sophistication will also introduce moreconstrained specifications that could be obstacles for the creativity and the 
imaginative extensions - related to the open world assumption — that are attractive in the different kinds of Web. It seems possible to deal with those difficulties by using the process of ontological development not only in an upward manner, from the coarser-grained levels to the more sophisticated ones, but also in a downward manner, coming back from explicitationto indistinct ontological stages - a way of being again more liberal with the definition of ontological categories - as all these levels have their own ontological relevance.

But here the purposes of philosophy and the Web diverge, since the philosophical return to the first levels of explicitation is at the same time a backward move from sophisticated propositions to the most basic and fundamental entities, while the same move within the Web framework would be a way to relax unduly restrictive formal constraints and to open fresh potentialities still not explored by users.

Pierre.Livet@univ-amu.fr

\section{References}

Frege, Gottlob. 1984. Collected Papers on Mathematics. Edited by Brian

McGuinness. Oxford: Blackwell.

Grenon, Pierre. 2003. "BFO (Basic Formal Ontology) in a Nutshell: A

Bi-CategorialAxiomatization of BFO and Comparison with Dolce,"

IFOMIS Report 06_2003.

Livet, Pierre, and Frédéric Nef. 2009. Les êtres sociaux. Paris: Hermann.

Monnin, Alexandre. 2012. "The Artifactualization of References and

'Substances' on the WEB," http://hal-paris1.archives-ouvertes.fr/hal-

$00610652 / \mathrm{fr} /$

Munn, Katherine, and Barry Smith (eds.). 2008. Applied Ontology: An

Introduction. Frankfurt: Ontos.

Smith, Barry. 1984. “Ontology (Science).” In Formal Ontology in Information

Systems, edited by CarolaEschenbach and Michael.Gruninger,

21-35.Proceedings of FOIS 2008. Amsterdam: ISO Press. 